\title{
Composición, abundancia y distribución de los sifonóforos (Cnidaria: Hydrozoa) en el Domo de Costa Rica, en febrero y marzo de 1979
}

\author{
Ma. Ana Fernández-Álamo ${ }^{1 *} \&$ Lidia Ramírez-Arriaga ${ }^{1}$ \\ 1. Laboratorio de Invertebrados, Facultad de Ciencias, Universidad Nacional Autónoma de México, Ciudad de México, \\ México; mafa@ciencias.unam.mx, lidiaram_57@yahoo.com.mx \\ * Correspondence
}

Recibido 30-I-2019. Corregido 01-VIII-2019. Aceptado 25-X-2019.

\begin{abstract}
Composition, abundance and distribution of siphonophores (Cnidaria: Hydrozoa) in the Costa Rica Dome, in February and March of 1979. Introduction: Costa Rica Dome is considered as a place with great importance, characterized by an upwelling event with high primary productivity. Siphonophores are predatory organisms that constitute one of the most abundant groups of the zooplankton biomass. Objective: To analyze the specific composition, abundance and distribution of siphonophores from the upwelling region of the central America. Methods: Samples were obtained during the oceanographic campaign DOMO I, which was carried out during February and March of 1979 by the 'Laboratorio de Oceanografía Física del Instituto de Ciencias del Mar y Limnología de la Universidad Nacional Autónoma de México (UNAM)'. The zooplankton samples were collected with a standard CalCoFI net with $0.3 \mathrm{~mm}$ mesh, and then analyzed in the laboratory. Results: A total of 30 species were identified, of which 27 belong to the suborder Calycophorae and three to the suborder Physonectae. Eudoxoides mitra, Muggiaea atlantica, Chelophyes contorta and Lensia hotspur were dominant species. C. contorta was observed with a wide distribution in the study area occupying $85 \%$ of the stations. It is followed by Eudoxoides mitra (82\%), Agalma okeni (73\%), Muggiaea atlántica (64\%) and Abylopsis eschscholtzi (61\%). The poligastric stage of calycophores species was predominant and the eudoxie stage was only observed in Diphyes dispar, Eudoxoides mitra, Chelophyes appendiculata, Ceratocymba dentata, Abylopsis eschscholtzi, Abylopsis tetragona, Bassia bassensis and Enneagonum hyalinum. Conclusion: The high diversity and density of siphonophores in this research show their importance on the great productivity in the Costa Rica Dome.
\end{abstract}

Key words: siphonophores; composition; diversity; density; distribution; Costa Rica Dome.

Fernández-Álamo, M.A., \& Ramírez-Arriaga, L. (2020). Composición, abundancia y distribución de los sifonóforos (Cnidaria: Hydrozoa) en el Domo de Costa Rica, en febrero y marzo de 1979. Revista de Biología Tropical, 68(Supl. 1), S225-S237.

Los sifonóforos son cnidarios coloniales, polimórficos con un alto grado de especialización funcional (Harvell, 1994; Pugh, 1999; Siebert et al., 2015); son pelágicos y depredadores, de importancia relevante en los estudios de las cadenas tróficas marinas por su abundancia y porque se alimentan principalmente de copépodos, ostrácodos, anfípodos y moluscos, así como huevos y larvas de otros crustáceos y peces, entre otros (Bigelow, 1911,
1926; Alvariño, 1967a; Alvariño, 1981; Purcell, 1981; Mackie, Pugh, \& Purcell, 1987; Purcell, 1997; Båmstedt, Fosså, Martinussen, \& Fosshagen, 1998; Pugh, 1999; Hosia \& Båmstedt, 2008; Mapstone, 2009, 2014). Tienen una amplia distribución mundial, pero la mayoría de las especies habitan en el cinturón trópico-ecuatorial oceánico (Bigelow, 1911; Alvariño 1971, 1981; Pugh, 1975; Purcell, 1997). Además, estos organismos se pueden 
reproducir rápidamente (Licandro, Souissi, Ibañez, \& Carré, 2012).

Dentro de los estudios más relevantes sobre los sifonóforos destacan los de Bigelow (1911), quien hace una excelente monografía del grupo con muestras tomadas por el "Albatross" en una amplía región del Pacífico Tropical Oriental, incluyendo algunas localidades oceánicas cercanas a la Isla Coco y principalmente estaciones costeras de los países de Centro América, donde se ubica el área de surgencia de Costa Rica. Alvariño (1971) hace una cuidadosa revisión de los trabajos de sifonóforos en el Pacífico proporcionando excelente información de las especies que han sido registradas en las aguas del Pacífico centroamericano, entre ellos sus artículos de 1969a y 1974. Gasca \& Suárez-Morales (1992) describen los sifonóforos recolectados durante la campaña oceanográfica DOMO III en noviembre de 1981 y Vicencio-Aguilar \& Fernández-Álamo (1996) enlistan 45 especies determinadas de las muestras recolectadas de los cruceros DOMO I, II, III y IV. Rodríguez-Saénz \& Gasca (2009) proporcionan una lista de especies de estos cnidarios en las aguas de Costa Rica, tanto del Mar Caribe como del Pacífico.

El propósito de este trabajo es contribuir al conocimiento de la composición específica, la densidad, la distribución y la caracterización ecológica de los sifonóforos que habitan las aguas de Domo de Costa Rica, durante los meses de febrero y marzo de 1979, correspondiente a la campaña oceanográfico Domo I.

\section{MATERIALES Y MÉTODOS}

El Domo de Costa Rica, situado en el Pacífico Tropical Oriental, es una región de intenso afloramiento de aguas profundas hacia la superficie, donde existen las condiciones físico-químicas para la fotosíntesis y con la elevada concentración de nutrientes que llevan permiten un gran desarrollo de la productividad primaria. Con ello se incrementa toda la compleja red alimentaria, trayendo para la región una intensa riqueza en la biodiversidad y una importancia económica sobresaliente en la producción pesquera (Fiedler, 2002; Fiedler \& Talley, 2006; Ballestero, 1999; Wehrtmann \& Córtes, 2009; Wehrtmann, Córtes, \& Echeverría-Saénz, 2009; Jiménez, 2016).

El material zooplanctónico fue recolectado durante el crucero oceanográfico Domo I a bordo del B/O "Mariano Matamoros", que se realizó durante febrero y marzo de 1979; se hicieron 37 arrastres de zooplancton. Los muestreos se realizaron utilizando una red estándar tipo CalCoFi con una malla de 0.3 $\mathrm{mm}$ mediante arrastres oblicuos en la zona epipelágica (0-200 m). Se colocó un contador de flujo TSK-946 en la boca de la red, previamente calibrado, con el fin de poder determinar el volumen de agua filtrada; los muestreos se incrementaron al detectar la zona de afloramiento del Domo de Costa Rica, de tal manera, que la mayor parte de las estaciones provienen del área comprendida entre los paralelos $7^{\circ}$ a $12^{\circ}$ de latitud Norte y $86^{\circ}$ a $92^{\circ}$ de longitud Oeste (Tabla 1).

Los parámetros físico-químicos como salinidad, oxígeno disuelto, temperatura y fluorescencia, fueron procesados en el Laboratorio de Oceanografía Física del Instituto de Ciencias del Mar y Limnología de la Universidad Nacional Autónoma de México y la información se encuentra en Barberán, Gallegos, Fernández, \& Meet (1985).

Los sifonóforos fueron separados de las muestras completas, con la ayuda de un microscopio de disección y pinzas de relojero; posteriormente fueron identificados empleando los trabajos de Sears (1953), Totton \& Barmann (1965), Alvariño (1981), Pugh (1999) y Mapstone (2014). El número total de organismos de las especies del suborden Calycophorae se determinó, tanto en la fase poligástrica (tomando en cuenta la cantidad más grande entre los nectóforos superiores e inferiores), como en la fase eudoxia (tomando en cuenta la cantidad más grande entre las brácteas y los gonóforos). Mientras que para los organismos del suborden Physonectae se consideraron sólo los pneumatóforos para su cuantificación (Ramírez-Arriaga, 1988). 
TABLA 1

Localización de las estaciones de muestreo en el Domo de Costa Rica durante la campaña oceanográfica DOMO I-1979

TABLE 1

Location of sampling stations in the Costa Rica Dome during the DOMO I-1979 oceanographic campaign

\begin{tabular}{|c|c|c|c|c|}
\hline Estación & Fecha & Hora local & Latitud N & Longitud W \\
\hline 1 & 05/II/1979 & $14: 10$ & $15^{\circ} 20^{\prime}$ & $95^{\circ} 04^{\prime}$ \\
\hline 3 & 06/II/1979 & 04:08 & $14^{\circ} 20^{\prime}$ & $94^{\circ} 59^{\prime}$ \\
\hline 4 & 06/II/1979 & $12: 15$ & $13^{\circ} 41^{\prime}$ & $94^{\circ} 56^{\prime}$ \\
\hline 6 & 07/II/1979 & 00:00 & $12^{\circ} 42^{\prime}$ & $95^{\circ} 02^{\prime}$ \\
\hline 7 & 07/II/1979 & 06:00 & $12^{\circ} 33^{\prime}$ & $95^{\circ} 07^{\prime}$ \\
\hline 15 & 09/II/1979 & 06:05 & $7^{\circ} 23^{\prime}$ & $94^{\circ} 58^{\prime}$ \\
\hline 18 & 10/II/1979 & 00:00 & $5^{\circ} 15^{\prime}$ & $94^{\circ} 57^{\prime}$ \\
\hline 19 & 10/II/1979 & $06: 15$ & $4^{\circ} 46^{\prime}$ & $95^{\circ} 00^{\prime}$ \\
\hline 21 & 10/II/1979 & $18: 05$ & $3^{\circ} 32^{\prime}$ & $95^{\circ} 00^{\prime}$ \\
\hline 22 & 10/II/1979 & $23: 50$ & $2^{\circ} 55^{\prime}$ & $94^{\circ} 59^{\prime}$ \\
\hline 23 & 11/II/1979 & 06:05 & $2^{\circ} 59^{\prime}$ & $94^{\circ} 32^{\prime}$ \\
\hline 25 & 11/II/1979 & $18: 05$ & $3^{\circ} 00^{\prime}$ & $93^{\circ} 46^{\prime}$ \\
\hline 26 & $12 / \mathrm{II} / 1979$ & 00:03 & $3^{\circ} 02^{\prime}$ & $93^{\circ} 14^{\prime}$ \\
\hline 28 & $12 / \mathrm{II} / 1979$ & 00:00 & $2^{\circ} 59^{\prime}$ & $92^{\circ} 10^{\prime}$ \\
\hline 30 & 13/II/1979 & $00: 15$ & $3^{\circ} 30^{\prime}$ & $91^{\circ} 27^{\prime}$ \\
\hline 31 & 13/II/1979 & 06:00 & $3^{\circ} 28^{\prime}$ & $90^{\circ} 51^{\prime}$ \\
\hline 33 & 13/II/1979 & $18: 10$ & $3^{\circ} 34^{\prime}$ & $89^{\circ} 35^{\prime}$ \\
\hline 37 & 14/II/1979 & $18: 10$ & $3^{\circ} 29^{\prime}$ & $87^{\circ} 13^{\prime}$ \\
\hline 42 & 15/II/1979 & $21: 10$ & $5^{\circ} 13^{\prime}$ & $85^{\circ} 57^{\prime}$ \\
\hline 43 & 16/II/1979 & $03: 15$ & $5^{\circ} 54^{\prime}$ & $85^{\circ} 55^{\prime}$ \\
\hline 44 & 16/II/1979 & 09:10 & $6^{\circ} 33^{\prime}$ & $86^{\circ} 02^{\prime}$ \\
\hline 47 & 17/II/1979 & $03: 15$ & $7^{\circ} 42^{\prime}$ & $85^{\circ} 59^{\prime}$ \\
\hline 48 & 17/II/1979 & $08: 52$ & $8^{\circ} 12^{\prime}$ & $85^{\circ} 58^{\prime}$ \\
\hline 50 & 17/II/1979 & $23: 55$ & $8^{\circ} 56^{\prime}$ & $86^{\circ} 21^{\prime}$ \\
\hline 58 & 19/II/1979 & $21: 05$ & $9^{\circ} 02^{\prime}$ & $91^{\circ} 12^{\prime}$ \\
\hline 74 & 23/II/1979 & $21: 05$ & $7^{\circ} 48^{\prime}$ & $88^{\circ} 59^{\prime}$ \\
\hline 76 & 24/II/1979 & 09:00 & $9^{\circ} 09^{\prime}$ & $89^{\circ} 01^{\prime}$ \\
\hline 85 & 25/II/1979 & $21: 05$ & $10^{\circ} 26^{\prime}$ & $88^{\circ} 01^{\prime}$ \\
\hline 91 & 26/II/1979 & $21: 10$ & $8^{\circ} 02^{\prime}$ & $87^{\circ} 42^{\prime}$ \\
\hline 95 & 27/II/1979 & $14: 12$ & $9^{\circ} 19^{\prime}$ & $86^{\circ} 56^{\prime}$ \\
\hline 97 & 27/II/1979 & $21: 05$ & $10^{\circ} 17^{\prime}$ & $86^{\circ} 56^{\prime}$ \\
\hline 104 & 28/II/1979 & $21: 05$ & $9^{\circ} 59^{\prime}$ & $87^{\circ} 24^{\prime}$ \\
\hline 110 & 1/III/1979 & $21: 00$ & $10^{\circ} 32^{\prime}$ & $89^{\circ} 57^{\prime}$ \\
\hline 112 & 2/III/1979 & 06:05 & $11^{\circ} 31^{\prime}$ & $89^{\circ} 58^{\prime}$ \\
\hline 117 & 3/III/1979 & 02:05 & $10^{\circ} 20^{\prime}$ & $91^{\circ} 02^{\prime}$ \\
\hline
\end{tabular}

Se calculó la densidad relativa de las especies y se expresó en número de org/ $1000 \mathrm{~m}^{3}$ de agua filtrada (Fig. 1). La distribución de las especies de sifonóforos se presenta en la Fig. 2 , en donde la densidad se proporciona en los siguientes intervalos de abundancia: escasa
(1-10), intermedia (11-100), abundante (101200) y muy abundante $(>201)$.

Para determinar la jerarquización ecológica de las especies se realizó la prueba de asociación de dos variables de OlmsteadTukey (Sokal \& Rohlf, 1995), que relaciona la 


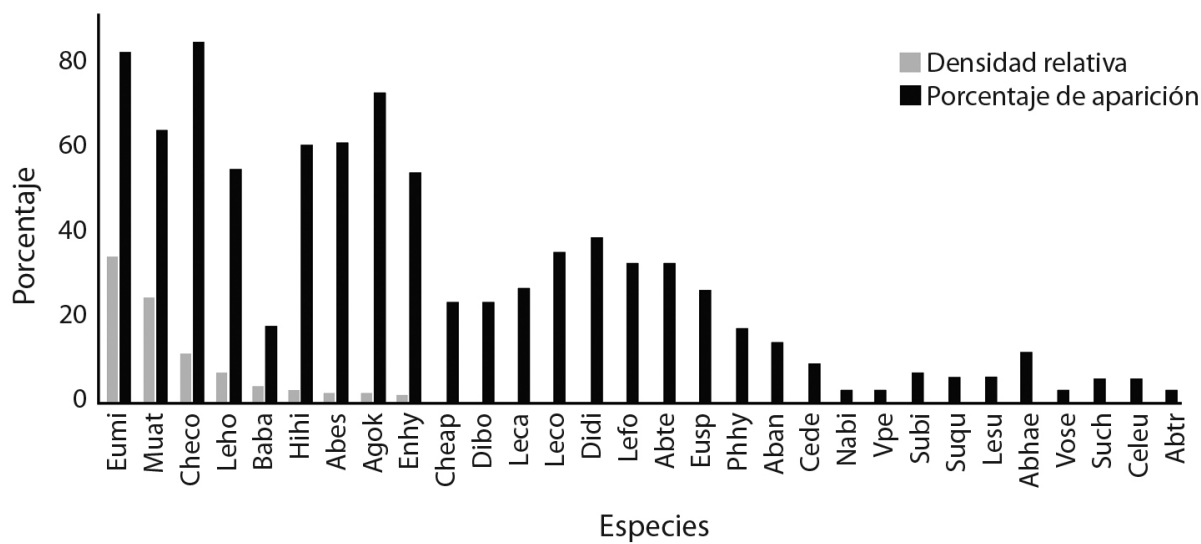

Fig. 1. Comparación entre la densidad relativa y el porcentaje de aparición de las especies durante la campaña oceanográfica DOMO I al Domo de Costa Rica durante febrero-marzo de 1979.

Fig. 1. Comparison between relative density and the percentage of appearance of the species during the oceanographic campaign DOMO I, Costa Rica Thermal Dome, February-March 1979.

densidad (org. $/ 1000 \mathrm{~m}^{3}$ ) con el porcentaje de su aparición en el área de estudio, proporcionando un gráfico sencillo.

Con la finalidad de identificar los patrones de distribución de las especies de sifonóforos, separando los estadios reproductivos o eudoxias de las formas poligástricas, se utilizó un análisis de agrupamiento de dos vías usando la abundancia estandarizada. Para disminuir la varianza se transformaron los valores a $\log (\mathrm{x}+1)$ y se utilizó el método de Sorensen (Bray-Curtis) con la distancia $\beta$ flexible -0.25 para conservar mejor los espacios de los grupos (McCune \& Grace, 2002). El análisis se realizó utilizando software PC-ORD 6 siguiendo las recomendaciones de McCune \& Grace (2002).

\section{RESULTADOS}

Se identificó un total de 30 especies, las cuales se enlistan siguiendo el arreglo taxonómico propuesto por Schuchert (2019).

Phylum Cnidaria Verril, 1865

Clase Hydrozoa Owen, 1843

Subclase Hydroidolina Collins, 2000

Orden Siphonophorae Eschscholtz, 1829

Suborden Calycophorae Leuckart, 1824

Familia Abylidae L. Agassis, 1862
Abyla trigona Quoy \& Gaimard, 1827

A. haeckeli Lens \& van Riemsdijk, 1908

*Abylopsis anomala (Sears, 1953)

A. eschscholtzii (Huxley, 1859)

A. tetragona (Otto, 1823)

Bassia bassensis (Quoy \& Gaimard, 1833)

Ceratocymba dentata (Bigelow, 1918)

C. leuckartii (Huxley, 1859)

Enneagonum hyalinum Quoy \& Gaimard, 1827

Familia Diphyidae Quoy \& Gaimard, 1827

Chelophyes appendiculata (Eschscholtz, 1829)

Ch. Contorta (Lens \& van Riemsdijk, 1908)

Diphyes bojani (Eschscholtz, 1825)

D. dispar Chamisso \& Eysenhardt, 1821

Eudoxoides mitra (Huxley, 1859)

E. spiralis (Bigelow, 1911)

Lensia campanella (Moser, 1917)

L. cossack Totton, 1941

L. fowleri (Bigellow, 1911)

L. hotspur Totton, 1941

L. subtilis (Chun, 1886)

Muggiaea atlantica Cunningham, 1892

Sulculeolaria biloba (Sars, 1846)

S. chuni (Lens \& van Riemsdijk, 1908)

S. quadrivalvis Blainville, 1830

Familia Hippopodiidae Kölliker, 1853

Hippopodius hippopus (Forsskål, 1776)

Vogtia pentacantha Kölliker, 1853

V. serrata (Moser, 1925) 


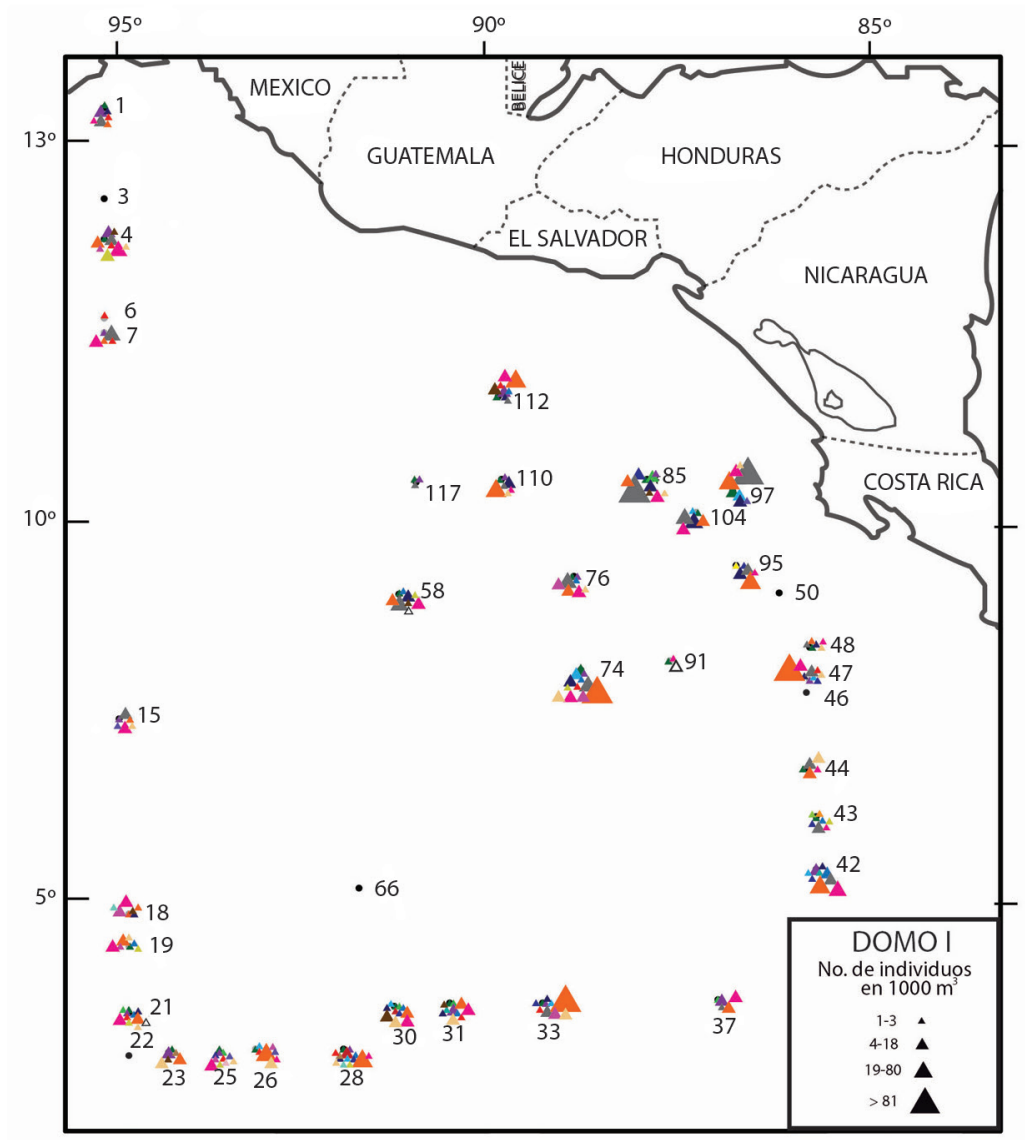

Especies de Siphonophora

Agalma oken
Nanomia bijuga
Physophora hydrostatica
Hippopodius hippopus
Vogtia serrata
Vogtia pentacantha
Sulculeolaria biloba
Sulculeolaria chuni
Sulculeolaria quadrivalvis

$\begin{array}{ll}\text { Lensia campanella } & \text { Eudoxoides mitra } \\ \text { Lensia cossack } & \text { Chelophyes appendiculata } \\ \text { Lensia fowleri } & \text { Chelophyes contorta } \\ \text { Lensia hotspur } & \text { Eudoxoides spiralis } \\ \text { Lensia subtilis } & \text { Abyla trigona } \\ \text { Muggiaea atlantica } & \text { Abyla haeckeli } \\ \text { Diphyes bojani } & \text { Ceratocymba dentata } \\ \text { Diphyes dispar } & \text { Ceratocymbaleuckarti } \\ & \text { Abylopsis eschscholtzi }\end{array}$

Fig. 2. Distribución y densidad de las especies de sifonóforos en el Domo de Costa Rica durante la campaña oceanográfica DOMO I, febrero-marzo de 1979.

Fig. 2. Distribution and density of siphonophores species in the Costa Rica Dome during the oceanographic campaign DOMO I, February-March 1979.

Suborden Physonectae Haeckel, 1888

Familia Agalmatidae Brand, 1834

Agalma okenii Eschscholtz, 1825

Nanomia bijuga (Delle Chiaje, 1841)

Familia Physophoridae Eschscholtz, 1829

Physophora hydrostatica Forsskål, 1775
*Taxón de validez incierta o discutida por diferentes expertos (taxon inquirendum) (Schuchert, 2019)

Del total de las 30 especies determinadas, el suborden Calycophorae mostró la mayor 
diversidad con 27, mientras que solo tres pertenecen a Physonectae, lo que coincide con los resultados en la mayoría de los trabajos sobre sifonóforos, por ejemplo, el de Lo \& Biggs (1996). De Calycophorae se identificaron tres familias, presentando Diphyidae la mayor riqueza con 15 especies, seguida por Abylidae con nueve y tres especies corresponden a la familia Hippopodiidae; mientras que en Physonectae, dos especies son de la familia Agalmatidae y una de la familia Physophoridae.

Las especies con mayor densidad relativa fueron los difidos Eudoxoides mitra, Muggiaea atlantica, Chelophyes contorta y Lensia hotspur (Fig. 1); en cuanto al porcentaje de aparición en la región de estudio, Chelophyes contorta ocupó el $85 \%$ de las estaciones de muestreo, seguida por E. mitra, M. atlántica, Abylopsis eschscholtzi y Agalma okeni se presentaron en el $82 \%, 73 \%, 64 \%$ y $61 \%$, respectivamente, perteneciendo las dos primeras a Diphydae, la tercera a Abylidae y la última al fisonéctido de la familia Agalmatidae (ver Lista taxonómica y Tabla 2).

Eudoxoides mitra presentó la densidad más alta en el área de estudio (2644 org./1000 m³),

TABLA 2

Sifonóforos en el Domo de Costa Rica durante la campaña oceanográfica DOMO I-1979

TABLE 2

Siphonophores Dome of Costa Rica during the DOMO I-1979 oceanographic campaign

\begin{tabular}{|c|c|c|c|c|c|}
\hline \multicolumn{2}{|l|}{ Taxa } & $\mathrm{D}$ & $\mathrm{D} \%$ & A & FA \\
\hline Eudoxoides mitra & [Eumi] & 2644 & 35 & 1621 & 82 \\
\hline Muggiaea atlantica & [Muat] & 1885 & 25 & 946 & 64 \\
\hline Chelophyes contorta & [Checo] & 905 & 12 & 557 & 85 \\
\hline Lensia hotspur & [Leho] & 544 & 7 & 275 & 55 \\
\hline Bassia bassensis & [Baba] & 265 & 3 & 144 & 18 \\
\hline Hippopodius hippopus & [Hihi] & 231 & 3 & 134 & 61 \\
\hline Abylopsis eschscholtzi & [Abes] & 188 & 2 & 116 & 61 \\
\hline Agalma okeni & [Agok] & 140 & 2 & 76 & 73 \\
\hline Enneagonum hyalinum & [Enhy] & 122 & 2 & 80 & 55 \\
\hline Chelophyes appendiculata & [Cheap] & 119 & 1 & 74 & 24 \\
\hline Diphyes bojani & [Dibo] & 83 & 1 & 54 & 24 \\
\hline Lensia campanella & [Leca] & 75 & 1 & 38 & 27 \\
\hline Lensia cossack & [Leco] & 62 & 1 & 44 & 36 \\
\hline Diphyes dispar & [Didi] & 67 & 1 & 40 & 39 \\
\hline Lensia fowleri & [Lefo] & 59 & 1 & 37 & 33 \\
\hline Abylopsis tetragona & [Abte] & 43 & 0.5 & 29 & 33 \\
\hline Eudoxoides spiralis & [Eusp] & 31 & 0.4 & 19 & 27 \\
\hline Physophora hydrostatica & [Phhy] & 29 & 0.3 & 20 & 18 \\
\hline Abylopsis anomala & [Aban] & 25 & 0.3 & 17 & 15 \\
\hline Ceratocymba dentata & [Cede] & 22 & 0.2 & 12 & 9 \\
\hline Nanomia bijuga & [Nabi] & 9 & 0.1 & 6 & 3 \\
\hline Vogtia pentacantha & [Vpe] & 10 & 0.1 & 4 & 3 \\
\hline Sulculeolaria biloba & [Subi] & 14 & 0.1 & 10 & 6 \\
\hline Sulculeolaria quadrivalvis & [Suqu] & 10 & 0.1 & 8 & 6 \\
\hline Lensia subtilis & [Lesu] & 11 & 0.1 & 8 & 6 \\
\hline Abyla haeckeli & [Abhae] & 12 & 0.1 & 6 & 12 \\
\hline Vogtia serrata & [Vose] & 7 & 0.09 & 5 & 3 \\
\hline Sulculeolaria chuni & [Such] & 4 & 0.05 & 2 & 6 \\
\hline Ceratocymba leuckarti & [Celeu] & 3 & 0.03 & 2 & 6 \\
\hline Abyla trigona & {$[\mathrm{Abtr}]$} & 1 & 0.01 & 1 & 3 \\
\hline Total & & & & 3533 & \\
\hline
\end{tabular}

$\mathrm{D}=$ Densidad, $\mathrm{D} \%=$ Densidad relativa, $\mathrm{A}=\mathrm{Abundancia}, \mathrm{FA}=$ frecuencia de aparición, [abreviatura de las especies de sifonóforos]. 
con valores altos de 407, 294, 282, en las localidades 37,74 y 47, posiblemente ubicadas sobre o cerca de la surgencia. También fue una especie dominante en el estudio de Lo \& Biggs (1996) en el Atlántico noroccidental cerca de Bermudas y en el Atlántico sur (Alvariño, 1981; Pugh, 1999). Es importante destacar que en general, la fase poligástrica fue la más abundante en las especies estudiadas y solo se observó la fase reproductora o eudoxia en Diphyes dispar, Eudoxoides mitra, Chelophyes appendiculata, Ceratocymba dentata, Abylopsis eschscholtzi, Abylopsis tetragona, Bassia bassensis y Enneagonum hyalinum.

De acuerdo a estos parámetros y aplicando la prueba de asociación de Olmstead-Tukey, la jerarquización ecológica de la comunidad de sifonóforos fue la siguiente: como especies dominantes quedaron Chelophyes contorta, Eudoxoides mitra, Agalma okeni, Muggiaea atlantica, Abylopsis eschscholtzi, Hippopodius hippopus, Lensia hotspur, y Enneagonum hyalinum. En la categoría de ocasionales se observaron a Diphyes dispar, Lensia campanella, Lensia fowleri, Lensia cossack, Chelophyes appendiculata, Diphyes bojani y Bassia bassensis, mientras que las 15 especies restantes quedaron en la categoría de raras (Fig. 3).

La Fig. 2 muestra la distribución y la densidad de las 30 especies de sifonofóros determinadas en este estudio, en el Pacífico centroamericano. Se puede apreciar que, en general, la mayor riqueza de especies y la mayor densidad de algunas de ellas se presentan en la región frente a las costas de El Salvador, Nicaragua y Costa Rica.

Mediante el análisis de agrupamiento de dos vías se definieron tres grupos de especies de sifonóforos $(12.5 \%$ de similitud) y tres grupos de estaciones $(25 \%$ y $18.5 \%$ de similitud). El grupo A conformado por las especies que presentaron sus mayores densidades en las estaciones influenciadas por la zona de surgencias; el grupo B estuvo formado por las especies que mantuvieron abundancias intermedias y con una mayor presencia de especies al margen de la región de surgencias. El grupo $\mathrm{C}$ estuvo constituido por especies raras y con baja frecuencia de aparición Fig. 4.

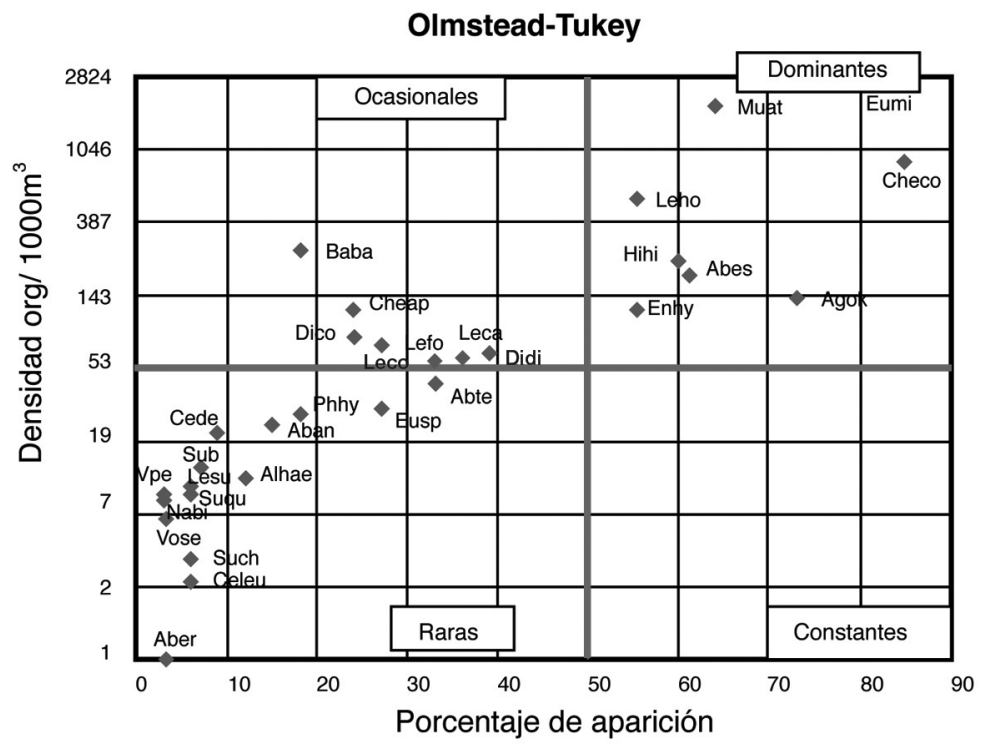

Fig. 3. Análisis de dominancia específica de los sifonóforos del Domo de Costa Rica durante febrero-marzo de 1979. Fig. 3. Analysis of the specific dominance of siphonophores in the Costa Rica Dome during February-March 1979. 


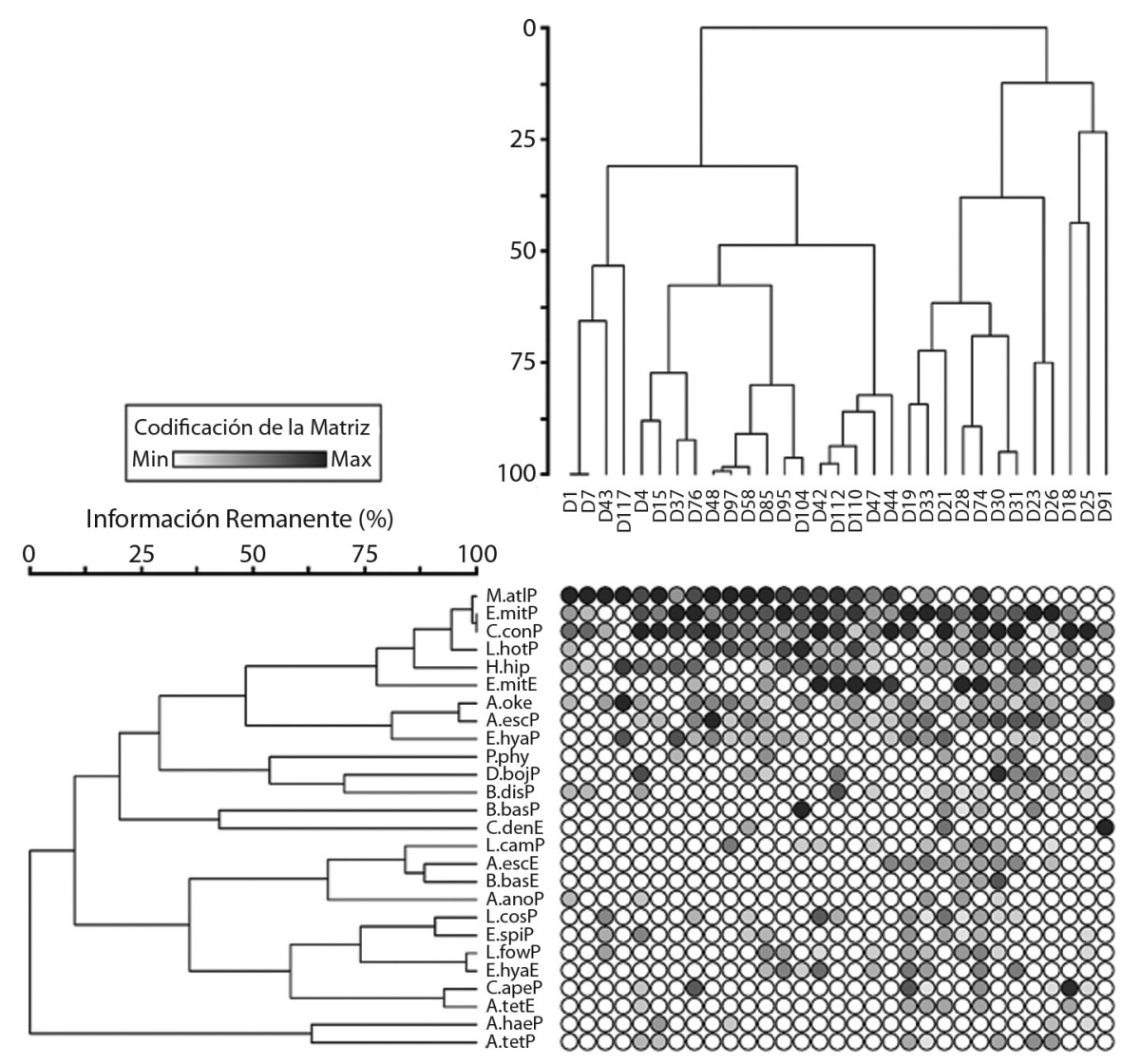

Fig. 4. Análisis de agrupamiento de dos vías de las especies de sifonóforos en el Domo de Costa Rica en febrero-marzo de 1979. $\mathrm{P}=$ fase poligástrica; $\mathrm{E}=$ fase eudoxia. Muggiaea atlantica (M.atlP), Eudoxoides mitra (E.mitP), Chelophyes contorta (C.conP), Lensia hospur (L.hotP), Hyppodius hippopus (H.hip), Eudoxoides mitra (E.mitE), Agalma okeni (A.oke), Abylopsis eschscholtzii (A.escP), Enneagonum hyalinum (E.hyaP), Physophora hydrostatica (P.phyP), Diphyes bojani (D.bojP), Diphyes dispar (D.disP), Bassia bassensis (B.basP), Chelophyes dentata (C.denE), Lensia campanella (L.camP), Abylopsis eschscholtzii (A.escE), Bassia bassensis (B.basE), Abylopsis anomala (A.anoP), Lensia cossak (L.cosP), Eudoxoides spiralis (E.spiP), Lensia fowleri (L.fowP), Enneagonum hyalinum (E.hyaE), Chelophyes appendiculata (C.apeP), Abylopsis tetragona (A.tetE), Abylopsis haeckeli (A.haeP), Abylopsis tetragona (A.tetP).

Fig. 4. Clustering analysis of two pathways of the siphonophores species in the Costa Rica Dome during February-March of 1979. $\mathrm{P}=$ polygastric phase; $\mathrm{E}=$ eudoxia phase. Muggiaea atlantica (M.atlP), Eudoxoides mitra (E.mitP), Chelophyes contorta (C.conP), Lensia hospur (L.hotP), Hyppodius hippopus (H.hip), Eudoxoides mitra (E.mitE), Agalma okeni (A.oke), Abylopsis eschscholtzii (A.escP), Enneagonum hyalinum (E.hyaP), Physophora hydrostatica (P.phyP), Diphyes bojani (D.bojP), Diphyes dispar (D.disP), Bassia bassensis (B.basP), Chelophyes dentata (C.denE), Lensia campanella (L.camP), Abylopsis eschscholtzii (A.escE), Bassia bassensis (B.basE), Abylopsis anomala (A.anoP), Lensia cossak (L.cosP), Eudoxoides spiralis (E.spiP), Lensia fowleri (L.fowP), Enneagonum hyalinum (E.hyaE), Chelophyes appendiculata (C.apeP), Abylopsis tetragona (A.tetE), Abylopsis haeckeli (A.haeP), Abylopsis tetragona (A.tetP).

\section{DISCUSIÓN}

Actualmente los estudios sobre sifonóforos se han incrementado demostrando su importancia en varios aspectos biológicos, ecológicos y filogenéticos (Mapstone, 2014; Munro et al.,
2018). Por lo tanto, el conocimiento sobre sifonóforos es esencial en los ecosistemas marinos $\mathrm{y}$ en particular constituyen una herramienta biológica importante en la comprensión de varios fenómenos oceanográficos. La información que aquí se presenta es importante 
para comprender mejor el Domo Térmico de Centro América.

En general, las 30 especies de sifonóforos determinadas en este trabajo tienen una amplia distribución en todos los océanos y mares del planeta y, de acuerdo a Ekman (1953) desde finales del siglo XIX varias de ellas se han usado en estudios zoogeográficos que ayudan a comprender diferentes aspectos de la oceanografía. En este sentido es interesante observar que Alvariño (1969b) describió que las especies Chelophyes contorta y C. appendiculata mostraron una distribución alopátrica, con la primera dominando en aguas cálidas y la segunda en aguas frías y por lo tanto eran indicadoras en la región del golfo de California. Alvariño (1974) lo que nota es una distribución y abundancia mayores de $C$. appendiculata en el Mar Caribe y una aparición escasa, limitada a pocas localidades en el Pacífico Centroamericano y lo opuesto sucede con $C$. contorta que justamente es lo que se observa en este trabajo y Pugh (1974) en su trabajo de Sifonóforos del Atlántico sur, solo la ubica en el suroeste de África en la región epipelágica. Moser (1925) incluye algunas de las especies registradas en el área de estudio, como Abylopsis eschscholtzii, Diphyes dispar y Physophora hydrostatica $\mathrm{y}$ las define como especies con distribución circumglobal, lo que es confirmado con los resultados obtenidos en este trabajo. En el Atlántico norte Pugh (1974) y Mackie et al. (1987) describen los aspectos zoogeográficos de las poblaciones de sifonóforos, mencionando que su distribución está influenciada por las masas de agua y por la posición de la termoclina, lo cual en general sucede en la mayoría de los integrantes del plancton, pero sin embargo no siempre está documentado.

Tomando en consideración los antecedentes de estos cnidarios coloniales en la región de estudio (Tabla 3), se encontró que de las especies definidas como dominantes en este trabajo, seis de ellas fueron registradas previamente en el área de estudio o en las regiones aledañas con ese mismo carácter, mientras que Lensia hospur y Enneagonium hylinun se observaron con una distribución muy aislada o con la categoría de raras (Alvariño, 1971; Gasca \& Suárez-Morales, 1992).

Sulculeolaria chuni apareció con una abundancia muy baja y una distribución restringida a solo dos estaciones y no fue citada por Alvariño (1971) en su listado de especies del Pacífico. De las especies con categoría de raras, tiene especial importancia $V$. serrata que no fue registrada en los trabajos de Bigelow (1911, 1931) y Alvariño (1969a, 1971, 1974). Sin embargo Alvariño (1967a) describe a $V$. kuruae como nueva especie que habita las aguas mesopelágicas en muestreos realizados frente a San Diego, California y vuelve a nombrarla en 1967 b y 1971. Schuchert (2019) actualmente la considera no válida y en sinonimia con $V$. serrata, por lo tanto la primera observación para las aguas del Domo fue la realizada por Vicencio-Aguilar \& Fernández-Álamo (1996) como V. kuruae. Bigelow \& Sears (1937) observaron a $V$. serrata a considerables profundidades (1066 a $2900 \mathrm{~m}$ ) en la Bahía de Cádiz, España y Moser (1925) considera que esta especie puede aparecer ocasionalmente en la superficie y la pregunta importante es si su presencia en el área de estudio se debe que fue sacada a la superficie por el afloramiento del Domo y por lo tanto puede ser considerada como especie indicadora de dicho fenómeno.

En general podemos resumir que el gran número de organismos y la gran diversidad de sifonóforos encontrada en este estudio en el Domo de Costa Rica, muestra la gran importancia que tienen dentro de la productividad secundaria y su relación con la presencia del Domo (Fig. 5). El Domo de Costa Rica es una zona de alta productividad primaria, que genera un aumento considerable en las comunidades de zooplancton, lo que se reflejó durante el presente estudio con el registro de altas concentraciones de sifonóforos en la parte central y occidental del Domo, entre los $9^{\circ}$ y $11^{\circ} \mathrm{N} \mathrm{y}$ entre $\operatorname{los} 85^{\circ}$ y $91^{\circ} \mathrm{W}$, confirmando los resultados obtenidos por De la Torre (1982) y Flores (1985), quienes registran los valores más altos de biomasa planctónica en esta región y se ha documentado que los sifonóforos tienen una 
TABLA 3

Estudios previos de los Sifonóforos en el Domo de Costa Rica y regiones adyacentes.

TABLE 3

Previous studies of the Siphonophores in the Dome of Costa Rica and adjacent regions.

\begin{tabular}{|c|c|c|}
\hline Especie & Localidad & Autor \\
\hline \multirow[t]{3}{*}{ Eudoxoides mitra } & Costas de Guatemala y Costa Rica y en la isla de Cocos & {$[\mathrm{Bi}]$} \\
\hline & Manzanillo, México hasta Panamá & Como Diphyopsis mitra $\left[\mathrm{Al}^{1}\right]$ \\
\hline & En las aguas del Domo de Costa Rica & {$[\mathrm{G} \& \mathrm{~S}],[\mathrm{V} \& \mathrm{~F}]$} \\
\hline \multirow[t]{3}{*}{ Muggiaea atlantica } & Costa de Guatemala & {$[\mathrm{Bi}]$} \\
\hline & Golfos de Panamá y Tehuantepec & {$\left[\mathrm{Al}^{1}\right]$} \\
\hline & En las aguas del Domo de Costa Rica & {$[\mathrm{G} \& \mathrm{~S}],[\mathrm{V} \& \mathrm{~F}]$} \\
\hline \multirow{3}{*}{ Chelophyes contorta } & Guatemala, Golfo de Panamá y Galápagos & {$[\mathrm{Bi}]$} \\
\hline & Manzanillo, México hasta Panamá & {$\left[\mathrm{Al}^{1}\right]$} \\
\hline & En las aguas del Domo de Costa Rica & {$[\mathrm{G} \& \mathrm{~S}],[\mathrm{V} \& \mathrm{~F}]$} \\
\hline \multirow[t]{3}{*}{ Hippopodius hippopus } & W plataforma de Albatross y Galápagos & {$[\mathrm{Bi}]$} \\
\hline & Manzanillo, México hasta Panamá & {$\left[\mathrm{Al}^{1}\right]$} \\
\hline & En las aguas del Domo de Costa Rica & {$[\mathrm{G} \& \mathrm{~S}],[\mathrm{V} \& \mathrm{~F}]$} \\
\hline \multirow[t]{3}{*}{ Abylopsis eschscholtzi } & Frente a Costa Rica & {$[\mathrm{Bi}]$} \\
\hline & Manzanillo, México hasta Panamá & {$\left[\mathrm{Al}^{1}\right]$} \\
\hline & En las aguas del Domo de Costa Rica & {$[\mathrm{G} \& \mathrm{~S}],[\mathrm{V} \& \mathrm{~F}]$} \\
\hline \multirow[t]{3}{*}{ Agalma okeni } & $\begin{array}{l}\text { Costa de Guatemala, Costa Rica, entre el Golfo de Panamá } \\
\text { y Galápagos y plataforma de Albatross }\end{array}$ & {$[\mathrm{Bi}]$} \\
\hline & Punta San Telmo, México hasta el Golfo de Panamá & {$\left[\mathrm{Al}^{1}\right]$} \\
\hline & En las aguas del Domo de Costa Rica & {$[\mathrm{G} \& \mathrm{~S}],[\mathrm{V} \& \mathrm{~F}]$} \\
\hline \multirow[t]{2}{*}{ Lensia hospur } & $\begin{array}{l}\text { En el Pacífico nororiental frente a las costas de California } \\
\text { y Baja California }\end{array}$ & {$\left[\mathrm{Al}^{2}\right]$} \\
\hline & En las aguas del Domo de Costa Rica & [G\&S], \\
\hline \multirow[t]{2}{*}{ Enneagonum hyalinum } & Guatemala, Golfo de Panamá, Galápagos y Perú & {$[\mathrm{Bi}]$} \\
\hline & En las aguas del Domo de Costa Rica & {$[\mathrm{G} \& \mathrm{~S}]$} \\
\hline
\end{tabular}

$\mathrm{Bi}=$ Bigelow (1911); $\mathrm{Al}^{1}$ y Al $\mathrm{Al}^{2}=$ Alvariño (1969a, 1971); G\&S = Gasca \& Suárez-Morales (1992); V\&F = Vicencio-Aguilar \& Fernández-Álamo (1996).

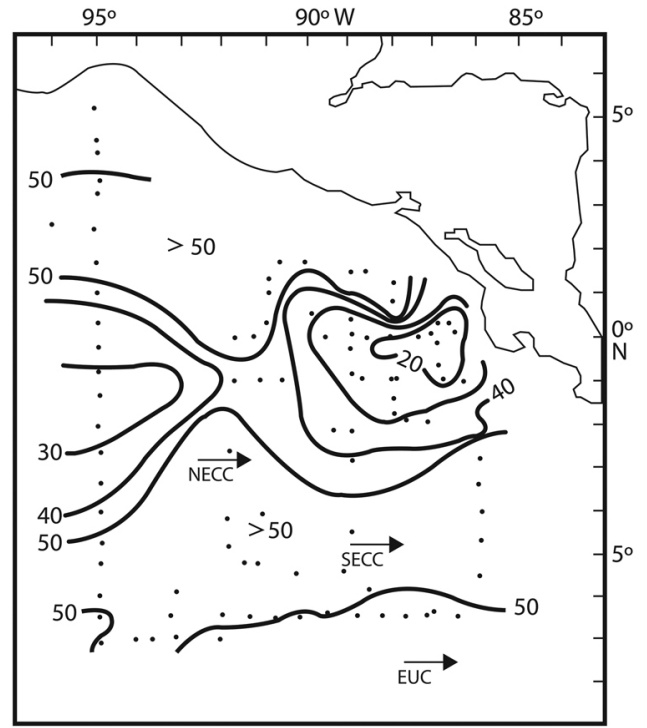

significativa contribución en la biomasa total del zooplancton (Robinson, 2004).

Declaración de ética: los autores declaran que todos están de acuerdo con esta publicación y que han hecho aportes que justifican su autoría; que no hay conflicto de interés de ningún tipo; y que han cumplido con todos los requisitos y procedimientos éticos y legales pertinentes. Todas las fuentes de financiamiento

Fig. 5. Distribución de las profundidades de la isoterma de los $20^{\circ} \mathrm{C}$ en el Domo de Costa Rica durante febrero-marzo de 1979. Modificado de Barberán, Gallegos, Fernández, \& Meet (datos sin publicar).

Fig. 5. Distribution of the $20{ }^{\circ} \mathrm{C}$ isotherm in the Costa Rica Dome, during February-March, 1979. Modified from Barberán, Gallegos, Fernández, \& Meet (unpublished data). 
se detallan plena y claramente en la sección de agradecimientos. El respectivo documento legal firmado se encuentra en los archivos de la revista.

\section{AGRADECIMIENTOS}

Se agradece la ayuda proporcionada por Adrián Fernández Álamo en la preparación de las figuras y la de Magali Roa Venicio en la elaboración de las tablas y el diagrama de relación entre la abundancia y la frecuencia de aparición de las especies estudiadas. Gracias a José Manuel Guerrero Ruiz por el apoyo en el análisis estadístico y a los dos revisores anónimos por sus acertadas sugerencias.

\section{RESUMEN}

Introducción: El Domo de Costa Rica es considerado como uno de los lugares de mayor importancia debido a que es una zona de afloramiento de agua, donde se desarrolla una alta productividad primaria. Los sifonóforos son organismos depredadores que constituyen uno de los grupos más abundantes de la biomasa zooplanctónica. Objetivo: Analizar la composición específica, la abundancia y la distribución de los sifonóforos. Métodos: Las muestras fueron obtenidas durante la campaña oceanográfica DOMO I realizadas en febrero y marzo de 1979 por el personal del Laboratorio de Oceanografía Física del Instituto de Ciencias del Mar y Limnología de la Universidad Nacional Autónoma de México (UNAM). Las muestras de zooplancton fueron recolectadas con una red estándar tipo CalCoFI elaborada con malla de $0.3 \mathrm{~mm}$ y analizadas en el Laboratorio de Invertebrados de la Facultad de Ciencias de la UNAM. Resultados: Se identificaron un total de 30 especies de las cuales 27 pertenecen al suborden Calycophorae y tres al suborden Physonectae. Eudoxoides mitra, Muggiaea atlantica, Chelophyes contorta y Lensia hotspur fueron las especies dominantes; C. Contorta mostró una amplía distribución con $85 \%$ de aparición, seguida por Eudoxoides mitra (82\%), Agalma okeni (73\%), Muggiaea atlántica (64\%) y Abylopsis eschscholtzi (61\%). La fase poligástrica fue predominante en las especies de calicóforos y la fase eudoxia solo fue observada en Diphyes dispar, Eudoxoides mitra, Chelophyes appendiculata, Ceratocymba dentata, Abylopsis eschscholtzi, Abylopsis tetragona, Bassia bassensis y Enneagonum hyalinum. Conclusión: La alta diversidad y densidad de los sifonóforos en esta investigación muestra su importancia en la gran productividad del Domo de Costa Rica.

Palabras clave: sifonóforos; composición; diversidad; densidad; distribución; domo térmico.

\section{REFERENCIAS}

Alvariño, A. (1967a). A new siphonophora, Vogtia kuruane n. sp. Pacific Science, 21(2), 236-240.

Alvariño, A. (1967b). Bathimetric distribution of Chaetognatha, Siphonophorae, Medusae and Ctenophorae of San Diego, California. Pacific Science, 21, 474-485

Alvariño, A. (1969a). Zooplankton from the Caribbean, Gulf of Mexico, mediate regions of the Pacific and fisheries. Abstracts (pp. 223-247). Proceedings of the IV Congress of Oceanography, Mexico.

Alvariño, A. (1969b). Zoogeografía del Mar de Cortés: Quetognatos y medusas. Anales del Instituto de Biologia, Universidad Nacional Autónoma de México, Series del Ciencias del Mar y Limnología, 40 (1), $11-54$.

Alvariño, A. (1971). Siphonophores of the Pacific with a review of the world distribution. Bulletin of the Scripps Institution of Oceanography, University of California, 16, 1-432.

Alvariño, A. (1974). Distribution of Siphonophores in the regions adjacent to the Suez and Panama canals. Fishery Bulletin, 72 (2), 527-546.

Alvariño, A. (1981). Siphonophorae. En D. Boltovskoy (Ed), Atlas del Zooplancton del Atlántico Sudoccidental (pp. 383-441). Mar de Plata, Argentina: Instituto Nacional de Investigación y Desarrollo Pesquero (INIDEP).

Båmstedt, U., Fosså, J. H., Martinussen, M. B., \& Fosshagen, A. (1998). Mass occurrence of the physonect siphonophore Apolemia uvaria (Lesueur) in Norwegian waters. Sarsia, 83, 79-85.

Ballestero, D., (1999). Remote sensing of vertically structured pigments. Tópicos Meteorológicos y Oceanográficos, 6 (2), 14-23.

Bigelow, H. B. (1911). Reports on the scientific results of the expedition to the eastern tropical Pacific, in charge of Alexander Agassiz, by the US Fish Commission Steamer "Albatross", from October 1904 to March 1905, Lieut. Commander L.M. Garrett, USN commanding. XXIII. The Siphonophorae. Memoirs of the Museum of Comparative Zoology, Harvard College, 38, 171-401.

Bigelow, H. B., (1926). Plankton of the offshore waters of the Gulf of Maine. Bulletin Bureau Commission Fisheries, 40(2) Doc. 968, 1-509.

Bigelow, H. B., (1931). Siphonophorae from the ARCTURUS Oceanographic Expedition. Zoologica, N. Y., $8(11), 525-592$.

Bigelow, H. B., \& Sears M. (1937). Siphonophorae. Report of Danish Oceanography Expedition 1908-1910, Medit., 2(H.2), 1-144. 
Córtes, J. (2009). A history of marine biodiversity scientific research in Costa Rica. En Wehrtmann, I. S., \& J. Cortés (Eds.), Marine Biodiversity of Costa Rica, Central America (pp. 47-80). Dordrecht: Springer Science + Business Media B.V.

De la Torre, Y. A. (1982). Distribución de los crustáceos decápodos planctónicos del Domo de Costa Rica. (Tesis de Licenciatura). Ciudad de México, México: Facultad de Ciencias, Universidad Nacional Autónoma de México.

Ekman, S. (1953). Zoogeography of the Sea. London: Sidwik and Jackson.

Fiedler, P. C. (2002). The annual cycle and biological effects of the Costa Rica Dome. Deep-Sea Research, $49,321-338$.

Fiedler, P. C. \& Talley, L. D. (2006): Hydrography of the eastern tropical Pacific: A review, Progress in Oceanography, 69, 143-180.

Flores, Z. M. (1985). Determinación y comparación de las biomasas totales y parciales del zooplancton obtenido durante el crucero Domo III en la región del Domo de Costa Rica. (Tesis de Licenciatura). Ciudad de México, México: Facultad de Ciencias, Universidad Nacional Autónoma de México.

Gasca, R., \& Suárez-Morales, E. (1992). Sifonóforos (Cnidaria: Siphonophora) del Domo de Costa Rica. Revista de Biología Tropical, 40, 125-130.

Harvell, C. D. (1994). The evolution of polymorphism in colonial invertebrates and social insects. The Quarterly Review of Biology, 96, 155-185.

Hosia, A., \& Båmstedt, U. (2008). Seasonal abundance and vertical distribution of siphonophores in western Norwegian fjords. Journal of Plankton Research, 30(8), 951-962.

Jiménez, J. A. (2016). El Domo Térmico de Costa Rica: Un oasis de productividad frente a las costas del Pacífico Centroamericano. San José, Costa Rica: Fundación MarViva.

Licandro, P., Souissi, S., Ibañez, F. \& Carré, C. (2012). Long-term variability and environmental preferences of calycophoran siphonophores in the Bay of Villefranche (north-western Mediterranean). Progress in Oceanography, 97-100, 152-163.

Lo, W., \& Biggs, D. C. (1996). Temporal varability in the nigth-time distribution of epipelagic siphonophores in the North Atlantic Ocean at Bermuda. Journal of Plankton Research, 18 (6), 923-939.

Moser, F. (1925). Die Siphonophoren der Deutschen Sud polar-Expedition 1901-03. Deutchen Sud polarExpedition, 17 (Zoology 9), 1-541.
Mackie, G. O., Pugh, P. R., \& Purcell, J. E. (1987). Siphonophore Biology. Advances in Marine Biology, 24, 97-262.

McCune, B. \& Grace, B. J. (2002). Analysis of Ecological Communities. Gleneden Beach, Oregon, U.S.A.: MjM Software Design.

Mapstone, G. M. (2009). Siphonophora (Cnidaria: Hydrozoa) of Canadian Pacific waters. Ottawa, Canada: National Research Council of Canada Research Press.

Mapstone, G. M. (2014). Global diversity and review of Siphonophorae (Cnidaria: Hydrozoa). PLOS ONE, 9(2), 1-37.

Munro, C., Siebert, S., Zapata, F., Howison, M., DamianSerrano, A., Samuel H. ... \& Dunn, C.W. (2018). Improved phylogenetic resolution within Siphonophora (Cnidaria) with implications for trait evolution. Molecular Phylogenetics and Evolution, 127, 823-833.

Pugh, P. R. (1974). The vertical distribution of the Siphonophores collected during the Sond Cruise, 1965. Journal of Marine Biology Association of the United Kingdom, 54, 25-90.

Pugh, P. R. (1975). The distribution of Siphonophores in a transect across the north Atlantic Ocean at $32^{\circ} \mathrm{N}$. Journal of Experimental Marine Biology and Ecology, 20, 77-97.

Pugh, P. R. (1999). Siphonophorae. En Boltvoskoy, D. (Ed.), South Atlantic Zooplankton (pp. 467-513). Leiden, Netherlands: Backhuys.

Purcell, J. E. (1981). Feeding ecology of Rhyzophysa eysenhardti a Siphonophore predator of fish larvae. Limnology and Oceanography, 26, 424-432.

Purcell, J. E. (1997). Pelagic cnidarians and ctenophores as predators: selective predation, feeding rates and effects on prey populations. Annales de l'Institute Océanographique de Paris, 73, 125-137.

Ramírez-Arriaga, L. (1988). Sistemática y distribución de los sifonóforos del Domo de Costa Rica. (Tesis de Licenciatura). Ciudad de México, México: Facultad de Ciencias, UNAM.

Robison, B. H. (2004). Deep pelagic biology. Journal of Experimental Marine Biology and Ecology, 300, 253-272

Rodríguez-Saénz, K., \& Gasca, R. (2009). Siphonophora. En Wehrtmann, I. S. \& J. Cortés (Eds). Marine Biodiversity of Costa Rica Central America (pp. 151-156). Dordrecht: Springer + Business Media B.V. 
Sears, M. (1953). Notes on siphonophores. 2. A revision of the Abylinae. Bulletin of the Museum of Comparative Zoology of Harvard College, 109, 1-119.

Siebert, S., Goetz, F., Church, S. H., Bhattacharyya, P., Zapata, F., Haddock, S. H. D., \& Dunn C. W. (2015). Stem cells in Nanomia bijuga (Siphonophora), a colonial animal with localized growth zones. EvoDevo, 6(22), 1-18

Schuchert, P. (2019). World Hydrozoa Database. Siphonophorae: World Register of Marine Species. Recuperado de http://www.marinespecies.org/aphia. php? $=$ taxdetails \&id=1371 on 2019-01-11.

Sokal, R. R., \& Rohlf, F. J. (1995). Biometry: The Principles and Practice of Statistics in Biological Research. 3rd Edition. New York: W.H. Freeman and Co.
Totton, A. K., \& Bargmann, E. (1965). A Synopsis of the Siphonophora. London: Trust British Museum (Natural History).

Vicencio-Aguilar, M., \& Fernández-Álamo, M. A. (1996). Zooplancton del Domo de Costa Rica: taxonomía y biogeografía. Revista de Biología Tropical, 44(2): 631-642.

Wehrtmann, I. S., \& Córtes, J. (2009). (Eds.) Marine Biodiversity of Costa Rica, Central America. Dordrecht: Springer Science + Business Media B.V.

Wehrtmann, I. S., Córtes, J., \& Echeverría-Saénz, S. (2009). Marine biodiversity of Costa Rica: Perspectives and conclusions. En Wehrtmann, I. S., \& J. Córtes (Eds.) Marine Biodiversity of Costa Rica, Central America (pp. 521-533). Dordrecht: Springer Science + Business Media B.V. 